\title{
Metaplastic Breast Carcinoma: A Rare Entity
}

\author{
Roshani Shrestha $^{*}$, Prakash Raj Neupane ${ }^{2}$, Bandana Satyal3 and Kamalesh Yadav3 \\ *Corresponding author: Roshani Shrestha \\ Address: ${ }^{1}$ Department of Medical Oncology, B\&B Hospital, Nepal; ${ }^{2}$ Department of Surgical Oncology, B\&B Hospital, \\ Nepal; 3Department of Pathology, B\&B Hospital, Nepal \\ E-mail $₫$ roshanishrestha2@gmail.com \\ Received: 30 Jun 2019; Accepted: o6 July 2019
}

\section{ABSTRACT}

Metaplastic Breast Carcinoma is a rare malignancy among invasive breast carcinoma. The patients usually present with large tumor size, axillary lymphnode metastasis and triple negative. The management of Metaplastic Breast carcinoma largely parallels that of Invasive Breast Carcinoma. In this case report, we found a case of metaplastic breast carcinoma with axillary lymphnode metastasis which is very rare.

Key Words: breast carcinoma; metastasis; lymphnode

\section{Introduction}

Invasive Breast carcinoma is the $3^{\text {rd }}$ leading cause of cancer in females of Nepal (Nepal-

Globocan, 2018). Among the invasive breast cancers, metaplastic breast cancer is a rare malignancy, comprising of $0.25-1 \%$. It is characterized by the histologic presence of two or more cellular types, commonly a mixture of epithelial and mesenchymal components (Oberman, 1987). The biomarker profile for MBC is usually triple negative (ER, PR and her2 neu). The World Health Organization (WHO) recognized MBC as a unique entity in 2000 (Pezzi et al., 2007). Even though it is a distinct entity, the optimal treatment strategies are unknown. So, management of MBC has largely paralleled that of Invasive Ductal Carcinoma (Shah et al., 2012).

\section{Case}

36 year old lady, unmarried had presented with bloody discharge from her left breast 1 year back. Serum prolactin was normal. Mammogram of bilateral breast showed normal reports with BIRADS score of 1 . After three months of duration she also noticed a lump in her left breast. Then she was evaluated with ultrasonography of bilateral breast which revealed a homogenous hypoechoic soft tissue mass with smooth margin of $19^{*} 17 \mathrm{~mm}$ in left periareolar region at 9' o clock position, with impression of benign lesion suggesting of fibroadenoma. FNAC of left breast lump showed features of Proliferative Breast disease without atypia with cystic changes. She was managed conservatively for fibroadenoma. But her lump progressively increased in size .Based on an excisional biopsy a metaplastic carcinoma was diagnosed. She proceeded with left Modified Radical Mastectomy. Pathology confirmed tumor dimension of $9 \mathrm{~cm}$, a high grade metaplastic 
carcinoma, Estrogen Receptor positive, progesterone receptor positive and her2 neu negative, stage pT3N1a. She is currently receiving adjuvant chemotherapy.

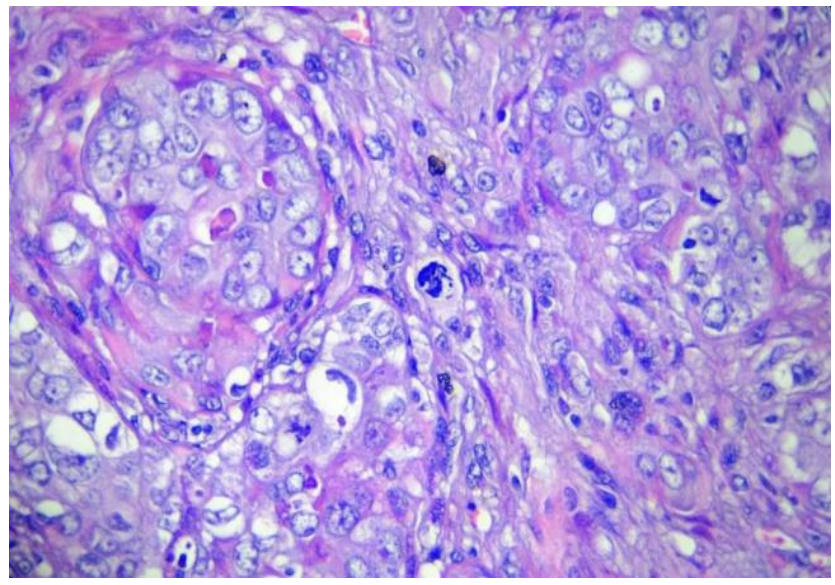

Figure 1: Tumor showing atypical cells and mitotic activity.

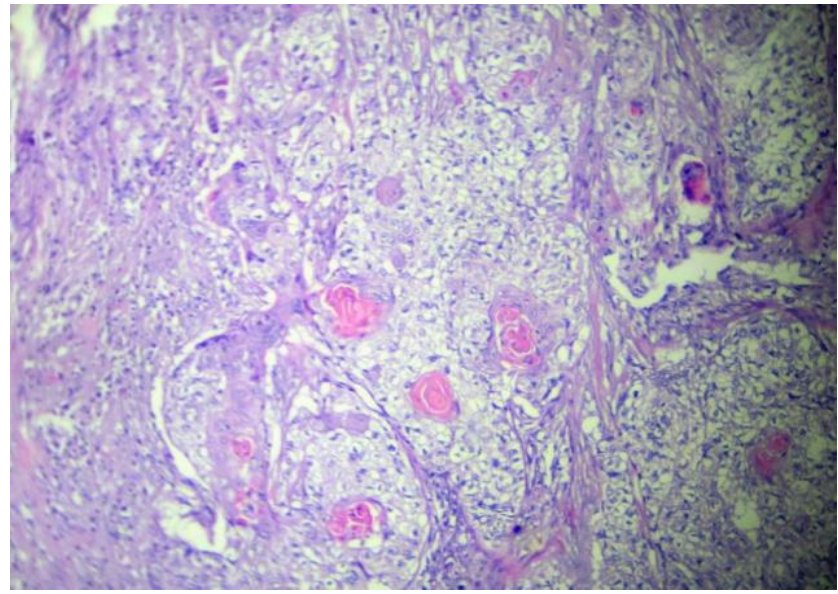

Figure 2: Tumor showing keratin perals.

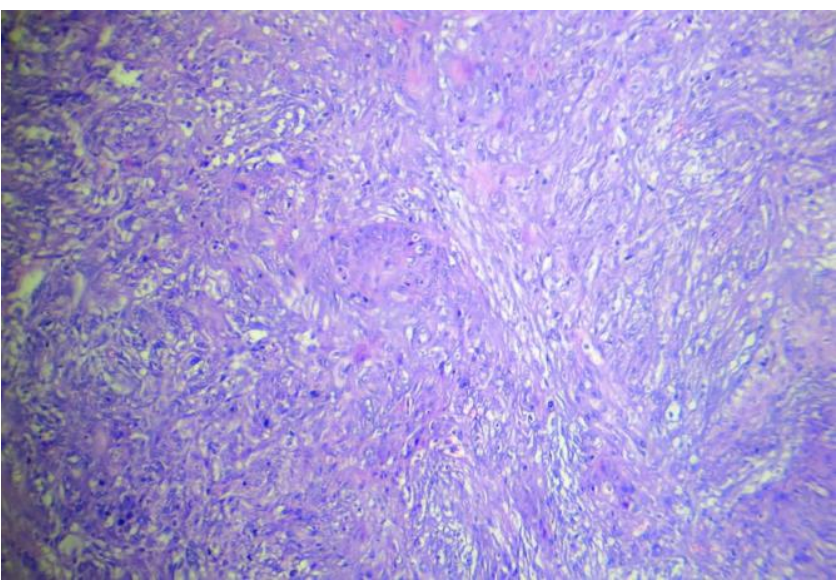

Figure 3: Mesenchymal components appears malignant with atypical clump to spindle cells having pleomorphic hyper chromatic nuclei and prominent nucleoli. 


\section{Discussion}

MBC was first described by Huvos et al., in 1973, was defined as a mammary carcinoma with mixed epithelial and sarcomatoid components (McKinnon and Xiao, 2015). The gold standard for diagnosing $\mathrm{MBC}$ is by pathologic assessment.

Patients with $\mathrm{MBC}$ were most commonly found to be older, with large tumor size, more advanced stage and usually triple negative (Lai et al., 2013). In this report the patient was of young age with large tumor size, axillary node positive and estrogen receptor positive. MBC usually presents with axillary lymph node metastasis in $22-31 \%$ of patients (Park et al., 2010). The patients with Lymphnode metastasis have a greater risk of developing metastatic disease and poorer prognosis than invasive Ductal carcinoma patients (Bae et al., 2011). As lymphnode positivity has poor survival, early diagnosis and treatment is crucial.

As typically $\mathrm{MBC}$ patients present with large tumors, they should undergo mastectomy rather than lumpectomy (Gibson et al., 2005). Adjuvant chemotherapy for MBC follows the treatment paradigm for conventional Invasive Ductal Carcinoma. In patients undergoing mastectomy, axillary nodal metastasis, large tumor size $>5 \mathrm{~cm}$ requires radiotherapy. But a study conducted by Luini et al. stated that radiotherapy is to be considered regardless of the type of surgery (Luini et al., 2007). In our patient, the patient will be proceeding to radiotherapy after adjuvant chemotherapy followed by hormonal therapy.

In conclusion, $\mathrm{MBC}$ is rare entity among breast carcinoma in Nepal, which is similar to reports of $\mathrm{MBC}$ from all over the world. In clinical therapy, $\mathrm{MBC}$ is usually follows the treatment paradigm for conventional Invasive Ductal carcinoma. The ideal treatment guideline for MBC is unknown and the potential predictors of treatment efficacy need to be explored.

\section{References}

Bae SY, Lee SK, Koo MY, Hur SM, Choi MY, Cho DH, Kim S, Choe JH, Lee JE, Kim JH, Kim JS. The prognoses of metaplastic breast cancer patients compared to those of triple-negative breast cancer patients. Breast cancer research and treatment 2011; 126(2): 471-478.

Gibson GR, Qian D, Ku JK, Lai LL. Metaplastic breast cancer: clinical features and outcomes. The American surgeon 2005; 71(9): 725-730.

Lai HW, Tseng LM, Chang TW, Kuo YL, Hsieh CM, Chen ST, Kuo SJ, Su CC, Chen DR. The prognostic significance of metaplastic carcinoma of the breast (MCB)-a case controlled comparison study with infiltrating ductal carcinoma. The Breast 2013; 22(5): 968-973.

Luini A, Aguilar M, Gatti G, Fasani R, Botteri E, Brito JA, Maisonneuve P, Vento AR, Viale G. Metaplastic carcinoma of the breast, an unusual disease with worse prognosis: the experience of the European Institute of Oncology and review of the literature. Breast cancer research and treatment 2007; 101(3): 349-353.

McKinnon E and Xiao P. Metaplastic carcinoma of the breast. Arch Pathol lab med 2015; 139: 819-822.

Nepal-Globocan, 2018. 
Global Journal of Cancer Case Reports | July 2019 | Volume 01| Issue 01 | Page 1-4

Oberman HA. Metaplastic carcinoma of the breast. A clinicopathologic study of 29 patients. The American journal of surgical pathology 1987; 11(12): 918-929.

Park HS, Park S, Kim JH, Lee JH, Choi SY, Park BW, Lee KS. Clinicopathologic features and outcomes of metaplastic breast carcinoma: comparison with invasive ductal carcinoma of the breast. Yonsei Medical Journal 2010; 51(6): 864-869.

Pezzi CM, Patel-Parekh L, Cole K, Franko J, Klimberg VS, Bland K. Characteristics and treatment of metaplastic breast cancer: analysis of 892 cases from the National Cancer Data Base. Annals of Surgical Oncology 2007; 14(1): 166173.

Shah DR, Tseng WH, Martinez SR. Treatment options for metaplastic breast cancer. ISRN oncology, 2012. 